\title{
Testing of Object-Oriented Programs Based on Finite State Machines*
}

\author{
Hyoung Seok Hong, Yong Rae Kwon and Sung Deok Cha \\ Department of Computer Science \\ Korea Advanced Institute of Science and Technology \\ 373-1, Kusong-dong, Yusong-gu, Taejon, Korea \\ \{hshong,yrkwon,cha\}@salmosa.kaist.ac.kr
}

\begin{abstract}
In object-oriented testing literature, a class is considered to be a basic unit of testing. A major characteristic of classes is the interaction between data members and member functions. This interaction is represented as definitions and uses of data members in member functions and can be properly modeled with finite state machines (FSM). In this paper, we discuss how FSMs can be effectively used for class testing. We demonstrate how to specify the behavior of classes using FSMs and present a test case generation technique based on FSMs. In our technique, FSMs are transformed into a flow graph from which we can explicitly identify data flows of the FSM. Then we generate test cases using conventional data flow testing techniques upon the flow graph.
\end{abstract}

\section{Introduction}

In object-oriented programs, there is a single entity called an object which represents both data and operations. Objects with similar data and operations can be specified by their common class. A class is a template that declares data members (attributes, properties) and operations (methods, member functions). A class is considered to be a basic unit of testing in object-oriented testing literature. The majority of class testing techniques generate test cases based on either algebraic specifications $[5,8,10]$ or model-based specifications $[13,14,21]$.

In this paper, we discuss the application of finite state machines (FSM) in class testing. A major characteristic of classes is the interaction between data members and member functions. The behavior of member functions is determined by the values of data

*This work is partially supported by the Korea Atomic Energy Research Institute under contract number KAERI/CM$103 / 94$. members and in turn, member functions manipulate data members. This interaction is represented as definitions and uses of data members in member functions and can be properly modeled with FSMs; data members represent the allowable states of an object and member functions represent a change of states, i.e., a transition of FSMs. Therefore, FSMs are often used as a specification of classes in most object-oriented analysis and design (OOA/OOD) methods[3, 6, 18, 19]. However, FSMs in OOA/OOD methods are described with English or pseudo-code and lack formal semantics to enable automated generation of test cases. We propose class state machine (CSM) for automatic test cases generation. The basic features of CSM are based on FSMs used in Rumbaugh et al.'s method[18]. CSM is a sort of extended FSMs composed of states, transitions, and data members of a class.

In our technique, we specify the behavior of classes in terms of CSM and generate test cases based on data flows in CSM. First, we transform CSM into a flow graph from which we can explicitly identify data flows; definitions and uses of each data member in CSM. Then we apply conventional data flow testing techniques $[9,16]$ upon the flow graph. This generates test cases which cover associations between definitions and uses of each data member. We can determine whether or not data flows in CSM are correctly implemented in the class's code with these test cases.

This paper is organized as follows: Section 2 discusses class specification based on FSM and formally defines CSM. Section 3 presents our testing technique hased on CSM. Section 4 describes related works to class testing and compares our work with them. Conclusion and future works are given in section 5 .

\section{Class Specification Based on FSMs}

Booch[3] discussed the motivation of using FSMs in class specification: An object has state, behavior, and identity. The existence of state within an object 
means that the order in which operations are invoked is important and we can formally characterize the behavior of the object in terms of FSMs.

Rumbaugh et al. [18] specified the behavior of a class in terms of states and events. When an event is received, the next state depends on the current state as well as the event; a change of state caused by an event is called a transition. The properties of states and transitions can be stated as follows:

- A state is an abstraction of the data members of a class. A set of values is grouped together into a state according to properties that affect the gross behavior of the class. Therefore, a state can be associated with the values of data members satisfying certain condition.

- A transition is composed of event, guard, and action. An event is an accident that cause the transition and can be represented as a call to a member function of the class. A guard is a condition that should be satisfied in order for the transition to occur and can refer to any data members in the class. An action is an operation that should be performed when the transition occurs and can refer to or manipulate the values of data members in the class.

For example, we assume that class Door has data members angle, locked and member functions open(), close(). If class Door has states, closed and opened, then each state can be associated with the values of data member angle as follows:

closed: angle $=0$

opened: $0<$ angle $\leq 180$

An event which triggers a transition can be represented as a call to a member function. For example, a transition from closed to opened is triggered when a member function open() is called. The guard and action of a transition can be defined in terms of data members. If a transition from state closed to opened can occur, a guard that the door is not locked should hold. This condition can be expressed in terms of data member locked as locked $=$ false. Similarly, the action of a transition from state opened to closed can be expressed by the assignment on data member angle as angle $:=0$.

\subsection{Class State Machine}

Definition 2.1 A class state machine (CSM) of a class $C$ is a tuple $M=(V, F, S, T)$ where

- $V$ is a finite set of data members of $C$.

- $F$ is a finite set of member functions of $C$.
- $S$ is a finite set of states, i.e., $S=\{s \mid s=($ de $f)\}$ where $d e f$ is a predicate on data members in $V$.

- $T$ is a finite set of transitions, i.e., $T=\{t \mid t=$ (source, target, fn, guard, action) $\}$ where

-- source $\in S$ is a state from which $t$ departs.

- target $\in S$ is a state at which $t$ arrives.

- $f n \in F$ is a member function which triggers $t$

- guard is a predicate on data members in $V$ and parameters of member functions in $F$.

- action is a set of computations on data members in $V$ and parameters of member functions in $F$.

For example, Fig. 1 shows a CSM of class Door.

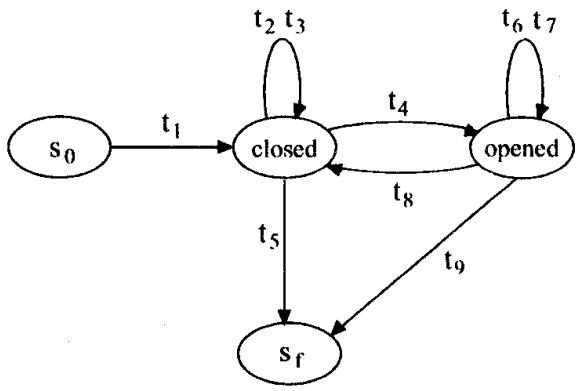

$$
\begin{aligned}
& V=\{\text { int angle, Boolean locked }\} \\
& F=\{\text { door }(), \sim \operatorname{door}(), \operatorname{lock}(\text { Boolean } 1), \text { angle(int a), } \\
& \text { open(int a), close(), move(int a) }\} \\
& S=\left\{s_{0}, \text { closed, opened, } s_{f}\right\} \\
& s_{0}=\text { (undefined), } \\
& s_{f}=\text { (undefined), } \\
& \text { closed }=(\text { angle }=0) \text {, } \\
& \text { opened }=(0<\text { angle } \leq 180) \\
& T=\left\{t_{i} \mid 1 \leq i \leq 11\right\} \\
& t_{1}=\left(s_{0}, \text { closed, door }(), \text { true, }\{\text { angle }:=0 \text {, }\right. \\
& \text { locked : = true }\} \text {, } \\
& \left.t_{2}=\text { (closed, closed, angle(a), true, }\{\mathrm{a}:=\text { angle }\}\right), \\
& \left.t_{3}=(\text { closed, closed, lock }(1) \text {, true, \{locked }:=1\}\right) \text {, } \\
& t_{4}=\text { (closed, opened, open }(a) \text {, locked }=\text { false } \wedge \\
& 0<\mathrm{a} \leq 180, \text { angle }:=\mathrm{a}\} \text { ), } \\
& t_{5}=\left(\text { closed, } s_{f}, \sim \text { door }(), \text { true, } \emptyset\right), \\
& t_{6}=\text { (opened, opened, angle(a), true, }\{\mathrm{a}:=\text { angle }\} \text { ), } \\
& t_{7}=\text { (opened, opened, move (a), } 0<\mathrm{a} \leq 180 \text {, } \\
& \text { \{angle }:=\mathrm{a}\} \text { ) } \\
& t_{8}=\text { (opened, closed, close(), true, }\{\text { angle }:=0\} \text { ), } \\
& \left.t_{9}=\text { (opened, } s_{f}, \sim \operatorname{door}() \text {, true, } \emptyset\right) \text {. }
\end{aligned}
$$

Figure 1: A class state machine for class Door 
A CSM of a class $C$ is composed of two parts: one representing data members and member functions of $C$; the other representing states and transitions of $C$. Class Door is composed of data members: angle, locked and member functions: door() which is a constructor; $\sim \operatorname{door}()$ which is a destructor; lock $(l)$ which assigns $l$ to locked; angle $(a)$ which assigns angle to $a$; open $(a)$, close (), move $(a)$ which manipulate angle.

Class Door has states: $s_{0}, s_{f}$, closed, and opened. Each state is composed of def which associates a state with a predicate which represents a set of values of data members. For example, closed.def is angle = 0 ; this means that when an object is in state closed, anlge $=0$ is true. There are special states in CSM, $s_{0}$ and $s_{f}$ :

- $s_{0}$ is an initial state representing the period before an object is created, i.e., a constructor of an object is called. $s_{0}$.def is undefined because data members have not yet been created.

- $s_{f}$ is a final state representing the period after an object is destroyed, i.e., a destructor of an object is called. $s_{f}$.def is undefined because data members are killed in $s_{f}$.

A transition consists of source, target, fn, guard, and action. If an object is in state source, $f n$ is called and guard is true, then a transition occurs. Hence, the pre-condition of a transition consists of source state and guard. When a transition occurs, action is performed and the state is changed into target. Hence, the post-condition of a transition consists of target state and action. For example, transition $t_{4}$ in Fig. 1 has closed, opened as a source and target state, respectively. $t_{4}$ can occur when an object is in closed, member function open $(a)$ is called and guard (locked $=$ false $\wedge 0<a \leq 180$ ) is true. When $t_{4}$ occurs, angle $:=a$ is performed and the state is changed into closed. Hence, the pre-condition of $t_{4}$ is $($ angle $=0) \wedge($ locked $=$ false $\wedge 0<a \leq 180)$. The post-condition of $t_{4}$ is $(0<$ angle $\leq 180) \wedge($ angle $:=a)$.

\subsection{Error transitions in CSM}

CSM in Fig. 1 is incompletely specified in the sense that some transitions are unspecified. For example, suppose that move $(a)$ is called when an object is in state closed. There exists no transition whose source state is closed and member function is move $(a)$. For another example, suppose that an object is in opened and move $(a)$ is called. If $0<a \leq 180$, tranistion $t_{7}$ occur. However, in the case of $a \leq 0 \vee a<180$ there exists no possible transition. We interprete these cases as an error transition, i.e., if move $(a)$ is called in closed, then the state of an object is changed to a state $s_{f} . s_{f}$ is an error state representing that an error has occured and $s_{c}$.de $f$ is undefined. In order to formally define error transitions, we adopt the foollowing notation.

Definition 2.2 Let $M=(V, F, S, T)$ be a CSM, $s$ $\in S$ be a state, and $f \in F$ be a member function. $T(s$, $f$ ) is a set of transitions whose source state is $s$ and triggering member function is $f$, i.e.,

$$
T(s, f)=\{t \in T \mid t . s o u r c e=s \wedge t . f n=f\} .
$$

In Fig. 1, T(closed, move $(a))$ is $\emptyset$ and T(opened, move $(a))$ is $\left\{t_{7}\right\}$.

Definition 2.3 Let $M=(V, F, S, T)$ be a CSM, $s$ $\in S$ be a state, and $f \in F$ be a member function.

- If $T(s, f)=\emptyset$, then $\left(s, s_{e}, f\right.$, true, $\left.\emptyset\right)$ is called an error transition of $M$.

- If $T(s, f)=\left\{t_{i} \mid 1 \leq i \leq n\right\}$ and $t_{1}$.guard $\vee$ $t_{2}$. guard $\vee \ldots \vee t_{n}$.guard $\neq$ true, then $\left(s, s_{e}, f\right.$, $\neg\left(t_{1}\right.$.guard $\vee t_{2}$.guard $\vee \ldots \vee t_{n}$.guard $\left.), \emptyset\right)$ is called an error transition of $M$.

For example, in Fig. 1 (closed, $s_{e}$, move (a), true, 0 ) is an error transition because $T($ closed, move $(a))=$ $\emptyset$. (opened, $s_{e}$, move $\left.(a), \neg(0<a<180), \emptyset\right)$ is also an error transition because $T$ (opene $\bar{d}$, move $(a)$ ) is $\left\{t_{7}\right\}$ and $t_{7}$.guard $\neq$ true. Fig. 2 shows a CSM with error transitions for class Door.

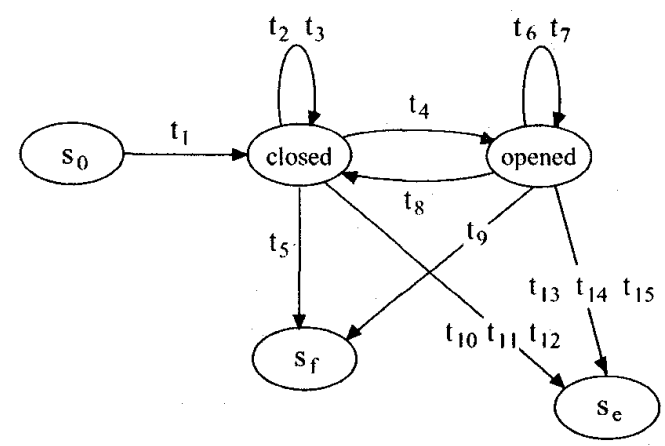

$t_{10}=\left(\right.$ closed, $s_{e}$, open ()$\left., \neg(0<\mathbf{a} \leq 180), \emptyset\right)$

$t_{11}=\left(\right.$ closed, $s_{e}, \operatorname{close}()$, true, $\left.\emptyset\right)$

$t_{12}=\left(\right.$ closed, $s_{e}$, move $($ a $)$, true, $\left.\emptyset\right)$

$t_{13}=$ (opened, $s_{e}, \operatorname{lock}(\mathrm{l})$, true, $\left.\emptyset\right)$

$t_{14}=$ (opened, $s_{e}$, open $(\mathrm{a})$, true, $\left.\emptyset\right)$

$t_{15}=$ (opened, $s_{\varepsilon}$, move $\left.(\mathrm{a}), \neg(0<\mathrm{a} \leq 180), \emptyset\right)$

Figure 2: A CSM with error transitions for class Door 


\section{Class Testing Based on FSMs}

In this section, we present a class testing technique based on CSM. Briefly our technique consists of the following steps:

1. We transform CSM into a flow graph called class flow graph (CFG) from which we can explicitly identify data flows in CSM.

2. We generate test cases from CFG using conventional data flow testing techniques.

(a) We identify definitions and uses of each data member in CFG.

(b) Based on these definitions and uses, every association between definitions and uses is established.

(c) Test cases are generated to cover these associations using data flow testing criteria. detail.

The following subsections describe these steps in

\subsection{Transforming CSM into CFG}

A flow graph is a graphical representation of a program's control structure. The nodes of a flow graph are statements of a program and edges indicate possible flow of control between nodes. In our technique, CSM is transformed into CFG. CFG is a flow graph which explicitly represent both the control and data flows of a class. The definition of CFG is as follows:

Definition 3.1 A class flow graph (CFG) of a class $C$ is a directed graph $G=(N, E)$ where

- $N=N_{s} \cup N_{g} \cup N_{t}$

- $N_{s}, N_{g}$, and $N_{t}$ are a set of $s$-nodes, $g$-nodes, and $t$-nodes, respectively.

- $E=E_{s t} \cup E_{s g} \cup E_{g t} \cup E_{t, s}$.

- $E_{s t}$ is a set of st-edges; a $s t$-edge is $(s, t), s$ $\in N_{s}, t \in N_{t}$

- $E_{s g}$ is a set of $s g$-edges; a $s g$-edge is $(s, g)$, $s \in N_{s}, g \in N_{g}$

- $E_{g t}$ is a set of $g t$-edge; a $g t$-edge is $(g, t), g$ $\in N_{g}, t \in N_{t}$

- $E_{t s}$ is a set of $t s$-edges; a $t s$-edge is $(t, s), t$ $\in N_{t}, s \in N_{s}$

A node of CFG is $s$-node, $g$-node, or $t$-node which represent state, guard of a transition, and member function in CSM, respectively. Edges of CFG represent possible flow of control between these nodes. We transform CSM into CFG as follows:
Algorithm 3.1 Transformation of CSM to CFG

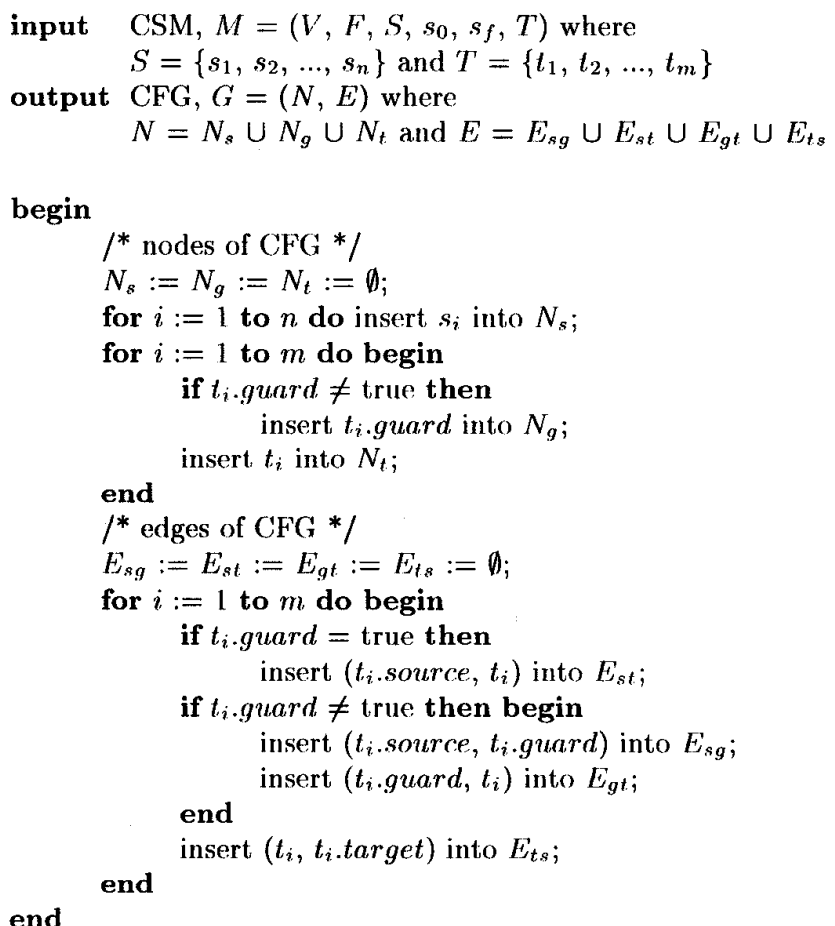

The above algorithm accepts a CSM as an input and outputs a CFG. For example, CSM in Fig. 1 is transformed into CFG in Fig. 3 by Algorithm 3.1.

Each state in CSM is represented by the corresponding $s$-node in CFG. In Fig. 3 there exist $s$-nodes, which are drawn by ellipses, representing states of CSM in Fig. $1 ; s_{0}, s_{f}, s_{e}$, closed, and opened. The diamond is a $g$-node; each guard of a transition in CSM which is not true is represented by a $g$-node. If the guard of a transition is true, there exists no $g$-node which represent the guard. The rectangle is a $t$-node; each transition in CSM is represented by a $t$-node.

Edges of CFG represent possible control flows between $s$-nodes, $g$-nodes, and $t$-nodes. We note that the following characteristics of the edges in CFG:

- In an st-edge $(s, t), s$ is source state of transition $t$; this represents that if an object is in $s$, transition $t$ will occur. For example, (closed, $t_{2}$ ) in Fig. 3 is an st-edge and represents that $t_{2}$ will occur in closed.

- An $s g$-edge $(s, g)$ is followed by an $g t$-edge $(g, t)$. $s$ is the source state of $t$ and $g$ is the guard of $t$; this represents that if an object is in $s$ and $g$ is satisfied, then $t$ will occur. For example, edges (closed, $\left.g_{4}\right)$ and $\left(g_{4}, t_{4}\right)$ represent that if an object is in closed and $g_{4}$ is satisfied, $t_{4}$ will occur. 


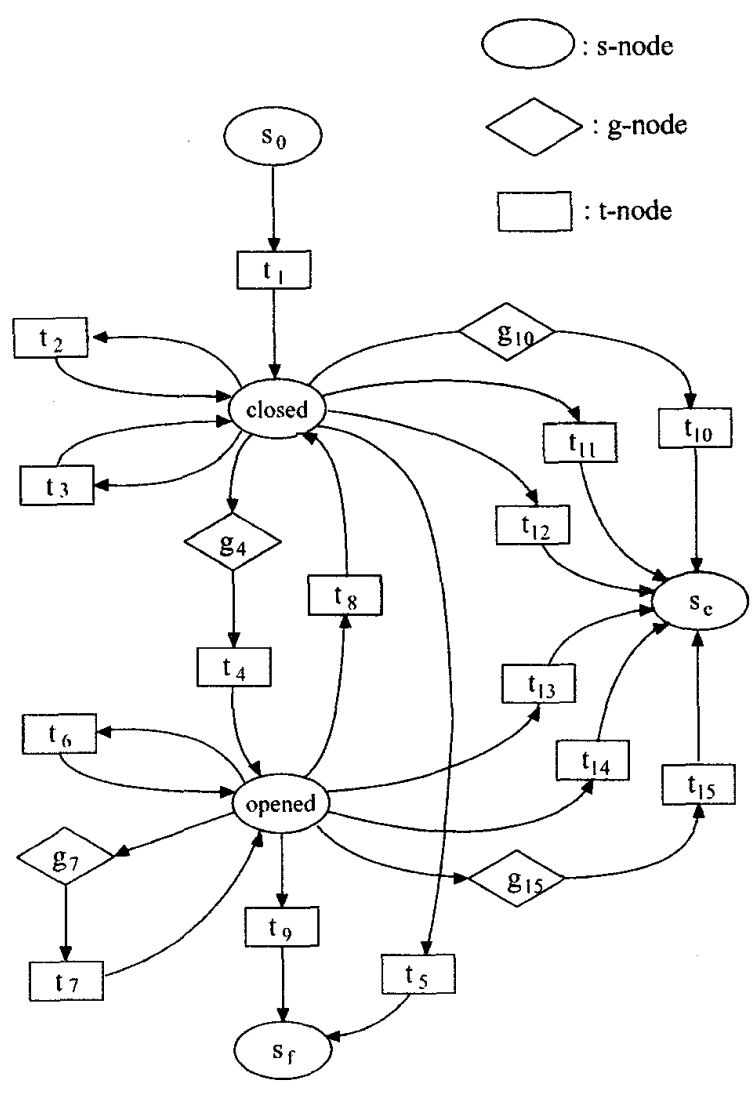

Figure 3: A class flow graph for class Door

- An $s t$-edge $(s, t)$ is followed by an $t s$-edge $\left(t, s^{\prime}\right)$ where $s^{\prime}$ is the target state of $t$. This edge represents that if $t$ occur, the state is changed into the target state of $t$. For example, edge $\left(t_{1}\right.$, closed $)$ represents that if $t_{1}$ occur, the state is changed into closed. Similarly, an $g t$-edge $(g, t)$ is followed by an $t s$-edge.

\subsection{Test Case Generations from CFG}

In our technique, test cases are generated using data flow testing techniques which selects test cases according to the locations of definitions and uses of variables in CFG.

First, each data member of a class is classified as being defined, computation-used (c-use) or predicateused (p-use) and this is identified by Rule 3.1. We do not consider definitions and uses of parameters of member functions, because we are interested in the interactions between data members and member functions.
Rule 3.1 Let $G=(N, E)$ be a CFG of a class $C$ and $v$ be a data member of $C$.

a) $v$ is said to be defined at $t$-node $t$ if taction assigns a value to $v$.

b) $v$ is said to be c-used at t-node $t$ if taction references $v$.

c) $v$ is said to be $p$-used at $s t$-edge $(s, t)$ if $s . d e f$ references $v$.

d) $v$ is said to be $p$-used at $s g$-edge $(s, g)$ if s.def references $v$.

e) $v$ is said to be $p$-used at $g t$-edge $(g, t)$ if $g$ references $v$.

In Rule 3.1 , a) and b) describe definitions and cuses of data members in CFG. For example, angle and locked are defined at $t$-node $t_{1}$ because $t_{1}$.action is $\{$ angle $:=0$, lock $:=$ true $\}$. And angle is c-used at $t$-node $t_{2}$ because $t_{2}$.action is $\{a:=$ angle $\}$.

In Rule $3.1, c), d$ ) and e) describe p-uses of data members in CFG. The pre-condition of a transition $t$ is t.source and t.guard, i.e., a transition $t$ can occur if an object is in t.source and t.guard is satisfied. Therefore, data members in (t.source).def and t.guard are p-used. For example, consider $t_{2}$ in Fig. 1. The source state of $t_{2}$ is closed and the guard of $t_{2}$ is true. Therefore, angle is p-used at st-edge (closed, $t_{2}$ ). The pre-condition of $t_{4}$ is (angle $=0$ ) and (lock $=$ false $\wedge 0<\mathrm{a} \leq 180)$. Hence, angle is p-used at $s g$-edge (closed, $\left.g_{4}\right)$ and lock is p-used at $g t$-edge $\left(g_{4}\right.$, $\left.t_{4}\right)$. Table 1 shows definitions and uses of each data member of class Door.

\begin{tabular}{|c||c|c|}
\hline & angle & lock \\
\hline \hline definition & $t_{1}, t_{4}, t_{7}, t_{8}$ & $t_{1}, t_{3}$ \\
\hline c-use & $t_{2}, t_{6}$ & $\emptyset$ \\
\hline p-use & $\left(\right.$ closed,$\left.t_{2}\right),\left(\right.$ closed,$\left.t_{3}\right)$, & $\left(g_{4}, t_{4}\right)$ \\
& $\left(\right.$ closed,$\left.g_{4}\right),\left(\right.$ closed,$\left.t_{5}\right)$, & \\
& $\left(\right.$ closed,$\left.g_{10}\right)$, (closed,$\left.t_{11}\right)$, & \\
& $\left(\right.$ closed,$\left.t_{12}\right)$, (opened,$\left.t_{6}\right)$, & \\
& (opened, $\left.g_{7}\right)$, (opened,$\left.t_{8}\right)$, & \\
& (opened, $\left.t_{9}\right)$, (opened, $\left.t_{13}\right)$, & \\
& (opened, $\left.t_{14}\right)$, (opened,$\left.g_{15}\right)$ & \\
\hline
\end{tabular}

Table 1: Definitions and uses of class Door

Second, based on definitions and uses of each data member we can establish associations between definitions and uses by Rule 3.2. Table 2 shows def-use associations of class Door.

Rule 3.2 Let $G=(N, E)$ be a CFG of a class $C$ and $v$ be a data member of $C$. 


\begin{tabular}{|c|c|c|}
\hline & angle & lock \\
\hline $\begin{array}{c}\text { def-c-use } \\
\text { association }\end{array}$ & $\begin{array}{c}\left(t_{1}, t_{2}\right),\left(t_{8}, t_{2}\right) \\
\left(t_{4}, t_{6}\right),\left(t_{7}, t_{6}\right)\end{array}$ & $\bar{\emptyset}$ \\
\hline $\begin{array}{l}\text { def-p-use } \\
\text { association }\end{array}$ & $\begin{array}{c}\left(t_{1}, \text { closed }, t_{2}\right),\left(t_{1}, \text { closed }, t_{3}\right),\left(t_{1}, \text { closed }, g_{4}\right),\left(t_{1}, \text { closed }, t_{5}\right), \\
\left(t_{1}, \text { closed }, g_{10}\right),\left(t_{1}, \text { closed }, t_{11}\right),\left(t_{1}, \text { closed }, t_{12}\right), \\
\left(t_{4}, \text { opened }, t_{6}\right),\left(t_{4}, \text { opened }, g_{7}\right),\left(t_{4}, \text { opened }, t_{8}\right),\left(t_{4}, \text { opened }, t_{9}\right), \\
\left(t_{4}, \text { opened }, t_{13}\right),\left(t_{4}, \text { opened }, t_{14}\right),\left(t_{4}, \text { opened }, g_{15}\right), \\
\left(t_{7}, \text { opened }, t_{6}\right),\left(t_{7}, \text { opened }, g_{7}\right),\left(t_{7}, \text { opened }, t_{8}\right),\left(t_{7}, \text { opened }, t_{9}\right), \\
\left(t_{7}, \text { opened }, t_{13}\right),\left(t_{7}, \text { opened }, t_{14}\right),\left(t_{7}, \text { opened }, g_{15}\right), \\
\left(t_{8}, \text { closed }, t_{2}\right),\left(t_{8}, \text { closed }, t_{3}\right),\left(t_{8}, \text { closed }, g_{4}\right),\left(t_{8}, \text { closed }, t_{5}\right), \\
\left(t_{8}, \text { closed }, g_{10}\right),\left(t_{8}, \text { closed }, t_{11}\right),\left(t_{8}, \text { closed }, t_{12}\right)\end{array}$ & $\begin{array}{r}\left(t_{1}, g_{4}, t_{4}\right) \\
\left(t_{4}, g_{4}, t_{4}\right)\end{array}$ \\
\hline
\end{tabular}

Table 2: Def-use associations of class Door

a) A def-c-use association of $v$ is an ordered pair ( $d$, c) where $d$ is a $t$-node containing a definition of $v$ and $c$ is a $t$-node containing a $c$-use of $v$ that can be reached by $d$.

b) A def-p-use association of $v$ is an ordered pair ( $d$, $p$ ) where $d$ is a $t$-node containing a definition of $v$ and $p$ is an $s t$-edge, $s g$-edge or $g t$-edge containing a p-use of $v$ that can be reached by $d$.

c) A def-use association is either def-c-use association or def-p-use association.

Finally, test cases are generated to cover associations between definitions and uses of each data member using certain data flow testing criteria. A number of coverage criteria such as all-definition, alluse, and all-du path coverage have been studied and compared $[9,16]$. All-definition coverage requires that every definition be covered by at least one use. Alluses coverage requires that at least one path from every definition to every use be exercised. All-du path coverage requires that every path from every definition to every use be exercised. For example, Table 3 shows test cases generated by all-def criteria for class Door.

$\left(s_{0}, t_{1}\right.$, closed,$t_{2}$, closed $\left., t_{5}, s_{f}\right)$

$\left(s_{0}, t_{1}\right.$, closed, $g_{4}, t_{4}$, opened, $t_{6}$, opened, $\left.t_{9}, s_{f}\right)$,

$\left(s_{0}, t_{1}\right.$, closed, $g_{4}, t_{4}$, opened, $g_{7}, t_{7}$, opened, $\left.t_{13}, s_{e}\right)$, $\left(s_{0}, t_{1}\right.$, closed $, g_{4}, t_{4}$, opened, $t_{8}$, closed $\left., t_{11}, s_{e}\right)$,

$\left(s_{0}, t_{1}\right.$, closed, $g_{4}, t_{4}$, opened, $\left.t_{9}, s_{f}\right)$,

$\left(s_{0}, t_{1}\right.$, closed,$t_{3}$, closed $, g_{4}, t_{4}$, opened, $\left.t_{9}, s_{f}\right)$

Table 3: Test cases generated by all-definition coverage

Each path in Table 3 represents a test case which covers a def-use association. For example, $\left(s_{0}, t_{1}\right.$, closed, $t_{2}$, closed, $\left.t_{5}, s_{f}\right)$ is a sequence of member functions, $(\operatorname{door}()$, angle $(a), \sim d o o r())$ and covers the association of definition of angle at $t_{1}$ and c-use at $t_{2}$.

\section{Related Works}

In general, FSM models of software abound in the testing literature[2]. Since Chow proposed testing techniques based on FSMs[7], many FSM-based testing techniques have been proposed especially in protocol conformance testing[4]. However, class testing based on FSMs is a relatively new concept. Recently, FSM-based class testing techniques have been proposed[15, 20] Turner and Robson[20] proposed a general testing procedure based on FSMs. Their technique determines input states and output states for each member function from the design of the class. However, this technique does not consider automatic test cases generation from FSMs. Kung et al.[15] proposed a class testing technique based on object state model (OSD) which is similar to statecharts[11]. They extract OSD from source code and generate test cases by constructing a spanning tree from OSD. Therefore, their technique mainly considers control flows in FSMs when generating test cases. In contrast, our technique generates test cases based on data flows in FSMs.

In object-oriented testing literature, a class is considered to be a basic unit of testing and several techniques have been proposed for class testing. Most of them is a specification-based technique using either algebraic specifications $[5,8,10]$ or model-based specifications $[13,14,21]$. An algebraic specification consists of signatures which define the syntactic properties and axioms which define the properties of member functions. A model-based specification specifies the pre-condition and post-condition of each member functions using well-defined mathematical models such as sets or sequences.

In $[5,8,10]$ test cases are generated as sequences 
of member functions based on the axioms in algebraic specifications. However, in algebraic specifications member functions are treated as a mathematical mapping without side-effects. Therefore, data flows between data members and member functions cannot be explicitly represented in these techniques. On the other hand, in $[13,14,21]$ test cases are generated based on the pre-condition and post-condition of each member function. Though member functions are tested whether or not they use and define data members correctly, data flows between member functions are not considered in these testing techniques.

There exist some techniques that adapt conventional flow graph-based testing techniques for class testing $[12,17,21]$. Zweben et al.[21] associated a flow graph with a class using model-based specifications. $A$ node in the flow graph represents a member function. An edge between node $A$ and $B$ means that it is permissible to invoke $A$ followed by $B$. Determining of whether or not an edge exists is based on the model-based specification of a class.

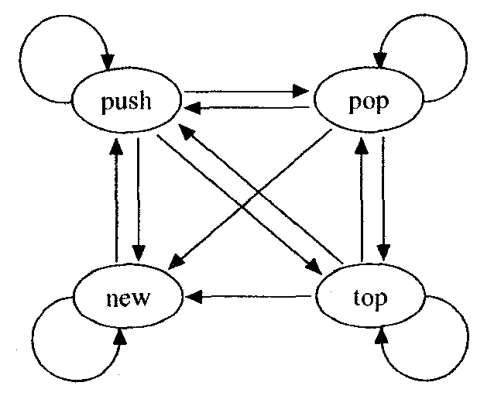

Figure 4: Zweben et al.'s flow graph for class Stack

For example, Fig. 4 shows a flow graph of class Stack constructed by Zweben et al.'s method. There exists no edge from new to pop, because new cannot be followed by pop. However, there exist infeasible paths such as (new, push, pop, pop) because states of a class are not considered when constructing the flow graph. In contrast, we transform FSMs into a flow graph and states of a class are considered in our flow graph. Therefore, all infeasible paths can be identified as a path containing an error transition.

Parrish et al.[17] extended Zweben et al.'s work so that a flow graph can be generated with or without specifications. A node of their flow graph is a member function and there exists an edge for every pair of nodes. Then a specification, if it exists, must be used to find infeasible paths. However, in the absence of specification, every sequence of member functions is assumed feasible.

Harrold and Rothermel[12] proposed data flow testing techniques for classes. They identify three levels of class testing: (i) intra-method testing which tests member functions individually, (ii) inter-method testing which tests a member function together with other member functions that it calls and, (iii) intra-class testing which tests the interactions of member functions when they are called in various sequences. To support each data flow in the three levels, they construct a flow graph which represents every possible sequences of member functions from the class's code. Then they generate test cases using inter-procedural data flow testing techniques.

The works in $[12,17]$ are code-based, i.e., a flow graph is constructed from the class's code without specifications and every sequence of member functions is assumed feasible. In contrast, our technique is specification-based, i.e., we specify the behavior of classes using FSMs and a flow graph is constructed from FSMs. There exist infeasible sequences of member functions in our flow graph; these sequences are determined by FSMs. Therefore, the works in $[12,17]$ and our work are complementary each other.

\section{Conclusion}

In this paper, we have proposed a testing technique for classes in object-oriented programs. Our technique is a specification-based testing using FSMs. By using FSMs as a specification of classes, we can properly model the interactions between data members and member functions. In order to test the behavior of classes effectively, we have generated test cases by transforming FSMs into a flow graph and applying data flow testing techniques upon the flow graph. We have used flow graphs instead of directly generating test cases from FSMs, hence we can adapt conventional data flow testing techniques for class testing.

Our work presented in this paper does not consider inter-class relationships. In general, there are three types of relationships between classes: association, aggregation and inheritance[18]. In several OOA/OOD methods such as $[6,18]$ these relationships are specified with extended FSMs similar to statecharts[11]. We are investigating testing techniques of inter-class relationships based on statecharts-like specifications.

\section{References}

[1] N. Amla and P. Ammann, "Using Z Specifications in Category Partition Testing," in Proceedings of the Seventh Annual Conference on Computer Assurance, Gaitherburg, 1992, pp. 3-10.

[2] B. Beizer, Software Testing Techniques, Van Nonstrand Reinhold, 1990. 
[3] G. Booch, Object Oriented Design with Applications, Benjamin Cummings, 1991.

[4] B. Bosik and M. ̈̈. Uyar, "Finite State Machine Based Formal Methods in Protocol Conformance Testing: from Theory to Implementation," Computer Networks and ISDN Systems, 22, 1991, pp. 7-33.

[5] L. Bouge, N. Choquet, L. Fribourg, and M.C. Gaudel, "Test Sets Generation from Algebraic Specifications Using Logic Programs," Journal of Systems and Software, 6, 1986, pp. 343-360.

[6] D. Champeaux, D. Lea, and P. Faure, Object Oriented System Development, Addison Wesley, 1993.

[7] T. Chow, "Testing Software Design Modeled by Finite-State Machines," IEEE Transactions on Software Engineering, Vol. SE-4, No. 3, May 1978, pp. 178-187.

[8] R. Doong and P.G Frankl, "Case Studies on Testing Object-Oriented Programs," in Proceedings of the 4th, Symposium, on Software Testing, Analysis and Verification, 1991, pp. 165-177.

[9] P.G. Frankl and E.J. Weyuker, "An Applicable Family of Data Flow Testing Criteria," IEEE Transactions on Software Engineering, Vol. 14, No. 10, Oct. 1988, pp. 1483-1498.

[10] J. Gannon, P. McMullin, and R. Hamlet, "DataAbstraction Implementation, Specification, and Testing," ACM Transactions on Programming Languages and Systems, Vol.31, No. 3, July 1981, pp. 211-223.

[11] D. Harel, "Statecharts: A Visual Formalism for Complex Systems," Science of Computer Programming, 8, 1987, pp. 231-274.

[12] M.J. Harrold and G. Rothermel, "Peforming Data Flow Testing on Classes," in Proceedings of the second ACM SIGSOFT Symposium on Foundations of Software Enginecring, Dec. 1994, pp. 154-163.

[13] I. Hayes, "Specification Directed Module Testing," IEEE Transactions on Softuare Engineering, Vol. SE-12, No. 1, Jan. 1986, pp. 124-133.

[14] X. Jia, "Model-Based Formal Specification Directed Testing of Abstract Data Types," in Proceedings of Computer Software and Applications Conference, 1993, pp. 360-366.

[15] D. Kung, N. Suchak, J. Gao, P. Hsia, Y. Toyoshima, and C. Chen, "On Object State Testing," in Proceedings of Computer Software and Applications Conference, 1994, pp. 222-227.

[16] S.C. Ntafos, "A Comparison of Some Structural Testing Strategies," IEEE Transactions on Software Engineering, Vol. 14, No. 6, June, 1988, pp. 868-874.
[17] A.S. Parrish, R.B. Borie, and D.W. Cordes, "Automated Flow Graph-Based Testing of ObjectOriented Software Modules," Journal of Systems and Softuare, 23, 1993, pp. 95-109.

[18] J. Rumbaugh, M. Blaha, W. Premerlani, F. Eddy, and W. Lorensen, Object Oriented Modeling and Design, Prentice Hall, 1991.

[19] S. Shlaer and S.J. Mellor, Object Lifecycles: Modeling the World in States, Prentice Hall, 1992.

[20] C.D. Turner and D.J. Robson, "The State-based Testing of Object-Oriented Programs," in Proceedings of Conference on Software Maintenance, 1993, pp. 302-310.

[21] S. Zweben, W. Heym, and J. Kimich, "Systematic Testing of Data Abstractions Based on Software Specifications," Journal of Software Testing, Verification, and Reliability, 1, 1992, pp. 39-55. 